\title{
TAUMOK - a Multi-Object Spectrograph for the Tautenburg Schmidt Telescope
}

\author{
H. Lehmann, R. Ziener, M. Ball \\ Thüringer Landessternwarte, Tautenburg, Germany \\ E. Pitz \\ Max Planck Institut für Astronomie, Heidelberg, Germany
}

\section{Introduction}

TAUMOK is a joint project of the Max Planck Institut für Astronomie Heidelberg and the Thüringer Landessternwarte Tautenburg. It is based on the concept of the "Spaltspinne" (Pitz 1993) now in operation at the Calar Alto Observatory. A first describtion of the technical concept was given by Pitz et al. (1993). TAUMOK was built at the MPIA Heidelberg in collaboration with astronomers and technicians from Tautenburg. It is now in the test phase and will have its first operation at the Tautenburg telescope in the middle of this year.

\section{Technical summary}

The use of a Schmidt telescope gives a very wide field for multi-object spectroscopy (TAUMOK will cover a field of $2.3^{\circ}$ ) but it also requires a series of special considerations. So, some of the details of the original "Spaltspinne" were modified to meet the site specific peculiarities. The size of the ground plate was minimized and yields a central obscuration of only $60 \mathrm{~cm}$ in diameter. The curved field of the Schmidt focus (deviation of up to $\pm 400 \mu \mathrm{m}$ from the mean focal plane) is flattened by a field lens - a solution alternative to the guiding of the fiber supporting rods on a sphere.

A processor-controlled positioner points 36 rods in the focal plane. Each of 34 of these rods carries two fibers with diameters of $100 \mu \mathrm{m}$, one fiber for the object and one for the sky background light. The opposite faces of the $8 \mathrm{~m}$ long fibers form the entrance slit of the spectrograph (modified UAGS from Carl Zeiss Jena) which is placed near the top of the fork mounting. At present four diffraction gratings are available providing reciprocal dispersions of 3.4, 10, 20 and $40 \mathrm{~nm} / \mathrm{mm}$. Object and background spectra are imaged on a CCD of $1152 \times 770$ pixels (pixel size $22.5 \mu \mathrm{m}$ ).

There are two new implementations for TAUMOK. First, on two rods two object fibers are replaced by image bundles of 6000 fibers each which feed a CCD detector (ST4 camera) for telescope guiding. Second, TAUMOK will have 36 sky background fibers adjacent to the object fibers and image bundles, respectively, in 40 arcsec distance on the same rod. This important improvement allows simultaneous sky background measurements. It became possible because of the 
larger entrance aperture $(1: 3)$ and the much smaller size of the fibers $(100 \mu \mathrm{m})$ compared to the "Spaltspinne".

Table 1. Basic technical data.

\begin{tabular}{ll}
\hline telescope & $1.34 \mathrm{~m}$ Schmidt \\
f-ratio & $1: 3$ \\
scale factor & $20 \mu \mathrm{m} /$ arcsec \\
field diameter & $160 \mathrm{~mm}=2.3^{\circ}$ \\
field flattening & field lens \\
diameter of ground plate & $60 \mathrm{~cm}$ \\
fibers & $34 \mathrm{object}+36$ sky backgound (Q+S AS100/140A) \\
distance object - background fibers & $40 \mathrm{arcsec}$ \\
fiber aperture & $100 \mu \mathrm{m}=5$ arcsec \\
pivot point radius & $135 \mathrm{~mm}$ \\
rod length & $210 \mathrm{~mm}$ \\
aquisition and guiding & TV guider fed by rods with image bundles \\
image bundle diameter & $500 \mu \mathrm{m}$ \\
comparison spectra & 36 fibers (Optran UV $300 / 360)$ fed by Th-A or He-A lamp \\
fat field & same fibers fed by quartz lamp \\
\hline \hline
\end{tabular}

\section{Further steps}

TAUMOK is now in the final test phase. It includes the determination of optimized parameters for the computer software controlling the positioning of the fiber supporting rods. So, a bending strain model is in hand which considers the deviation of the fiber tips from the calculated places in different telescope positions. Another problem is the handling of the $8 \mathrm{~m}$ long fibers. A laboratory arrangement had been made at Tautenburg for the measurement of the fiber degradation.

TAUMOK should be available for observations in the middle of 1994 . First astronomical projects will be the measurement of the redshifts of galaxies, the spectroscopical check of quasar candidates and the search for faint B stars in the galactic plane as indicators for a possible spiral structure behind the Perseus arm.

Acknowledgments. We are grateful to H. Elsässer and S. Marx for stimulating and supporting the project. Continuous support was given by $\mathrm{H}$. Benesch, H. Bellemann, B. Grimm, G. Hille, C. Marien, W. Rauh, K. Wagner, and K. Zimmermann from the MPIA Heidelberg, by H. Löchel and M. Pluto from the LSW Tautenburg, and by F. Kretschmer from the AIP Potsdam.

\section{References}

Pitz, E. 1993, in Fiber Optics in Astronomy II, P. M. Gray, A. S. P., Conference Series, Vol. 37, p. 20

Pitz, E., Lorenz, H., Elsässer, H. 1993, ibid., p. 166 